\title{
Stability of rotating states in a weakly-interacting Bose-Einstein condensate
}

\author{
G. M. Kavoulakis \\ Mathematical Physics, Lund Institute of Technology, P.O. Box 118, S-22100 Lund, Sweden
}

(October 28, 2018)

\begin{abstract}
We investigate the lowest state of a rotating, weakly-interacting Bose-Einstein condensate trapped in a harmonic confining potential that is driven by an infinitesimally asymmetric perturbation. Although in an axially-symmetric confining potential the gas has an axially-symmetric single-particle density distribution, we show that in the presence of the small asymmetric perturbation its lowest state is the one given by the mean-field approximation, which is a broken-symmetric state. We also estimate the rate of relaxation of angular momentum when the gas is no longer driven by the asymmetric perturbation and identify two regimes of "slow" and "fast" relaxation. States of certain symmetry are found to be more robust.
\end{abstract}

PACS numbers: 03.75.Fi, 05.30.Jp, 67.40.Db, 67.40.Vs

\section{INTRODUCTION}

A Bose-Einstein condensate of trapped alkali-metal atoms [1] is an interesting system for studying phenomena connected with superfluidity. The behavior of this system under rotation has been studied extensively. Experimentally vortex states in a two-component BoseEinstein condensate were observed by Matthews et al. [2]. Also, Madison et al. [3] and more recently Abo-Shaeer et al. [4] and Haljan et al. [5] have created vortices in a single-component condensate.

Theoretical studies of this problem have been performed in the limit of weak interactions between the atoms [6 16], as well as in the Thomas-Fermi limit of strong interactions 17 23]. Up to now most experiments are in the Thomas-Fermi regime; however in the recent experiment of Görlitz et al. 24] in a cigar-shaped trap, the limit of weak interactions was reached transversely to the long axis of the trap. In addition, in the experiment of Ref. [1], more than 100 vortices were created and as argued by Ho [16], in such a system one can easily get to the regime of weak interactions. In the present article we restrict ourselves to the limit of weak interactions.

As discussed by many authors [6 16] in this limit there is a degeneracy due to the harmonic confining potential, which corresponds to the many different ways of distributing $L$ units of angular momentum among $N$ atoms. The goal, therefore, is to incorporate the interactions which lift the degeneracy and determine the lowest-energy state of the system.

A basic question related to these systems concerns the lowest state for a given $L$ and $N$ in a symmetric confining potential. References [6 14] have dealt with this issue. Having understood the question of a symmetric confining potential, one can then examine the ground state in the presence of a realistic trap, which has a small but finite asymmetry, or even in a more deformed trap. Our main goal is to investigate (i) the single-particle density distribution of the condensate when it equilibrates in the rotating frame, and (ii) the characteristic timescale for relaxation of angular momentum of the condensate when the trap stops to rotate and the gas is no longer driven externally.

Our article is organized in the following way: We describe our model in Sec.II, examining how a small symmetry-breaking harmonic potential affects the lowest-energy state of a repulsive weakly-interacting Bose-Einstein condensate which is confined in a symmetric harmonic potential. In Sec. III we demonstrate that the state that minimizes the energy in the presence of a small asymmetric perturbation is the one given by the mean-field approximation, which is a broken-symmetric state. Finally, in Sec. IV we estimate the rate of relaxation of angular momentum when the asymmetric drive stops to rotate, and in Sec. V we summarize our results.

\section{MODEL}

\section{A. Symmetric confining potential}

Let us start with the Hamiltonian $H$, given by

$$
H=\sum_{i}\left[-\frac{\hbar^{2}}{2 M} \nabla_{i}^{2}+\frac{1}{2} M \omega_{\perp}^{2}\left(x_{i}^{2}+y_{i}^{2}\right)\right],
$$

with $M$ being the atomic mass. In what follows we assume that the cloud rotates around the $z$ axis, along which it remains in its ground state, so effectively we examine a two-dimensional problem. Let us assume for the moment that the trapping potential is that of an isotropic harmonic oscillator of frequency $\omega_{\perp}$ in the $x-y$ plane. For this potential the single-particle energies $\epsilon_{n_{r}, m}$ are given by

$$
\epsilon_{n_{r}, m}=\left(2 n_{r}+|m|+1\right) \hbar \omega_{\perp}
$$

where $n_{r}$ is the radial quantum number, and $m$ is the quantum number corresponding to the angular momentum. Let us also denote the corresponding single-particle states as $\phi_{n_{r}, m}$ (single-particle states are denoted with 
small initial letters and many-particle states are denoted with capital initial letters.) As we discuss in the following subsection, a crucial observation according to Eq. (2) is that there is a degeneracy between states with $\left(n_{r}=0\right.$; $\left.m=m_{0}\right),\left(n_{r}=1 ; m=m_{0}-2\right),\left(n_{r}=2 ; m=m_{0}-4\right)$, etc., with an energy $\left(m_{0}+1\right) \hbar \omega_{\perp}$. States with $m$ having the opposite sign as $L$ are excluded, since mixing them results in higher-energy $8,12,13$.

\section{B. Symmetry-breaking confining potential}

Let us now assume that in addition to the circularlysymmetric harmonic potential there is a small symmetrybreaking potential $\Delta H$ which has the form

$$
\Delta H=\sum_{i} \frac{\varepsilon}{2} M \omega_{\perp}^{2}\left(x_{i}^{2}-y_{i}^{2}\right),
$$

where $\varepsilon \ll 1$. The eigenvalues of the Hamiltonian $H+$ $\Delta H$ are known analytically in cartesian coordinates, and they are given by

$$
\epsilon_{n_{x}, n_{y}}=\left(n_{x} \sqrt{1+\varepsilon}+n_{y} \sqrt{1-\varepsilon}\right) \hbar \omega_{\perp},
$$

where $n_{x}$ and $n_{y}$ are the number of quanta of oscillation along the $x$ and $y$ directions respectively. The corresponding eigenfunctions are given by the product of the eigenstates along the $x$ and $y$ directions with $n_{x}$ and $n_{y}$ quanta, respectively,

$$
\phi_{n_{x}, n_{y}}(x, y)=\phi_{n_{x}}\left(x / a_{x}\right) \phi_{n_{y}}\left(y / a_{y}\right) .
$$

Here $a_{i}=\left(\hbar / M \omega_{i}\right)^{1 / 2}$, is the oscillator length along direction $i$, with $\omega_{x}, \omega_{y}=\omega_{\perp} \sqrt{1 \pm \varepsilon}$.

Although the non-interacting problem can be solved exactly, it is instructive to continue working in cylindrical polar coordinates, and consider $\Delta H$ as a small perturbation. In these coordinates $\Delta H$ links single-particle states which differ by two units of angular momentum, $m^{\prime}-m= \pm 2$. Therefore $\Delta H$ links the degenerate states with $\left(n_{r}=0 ; m=m_{0}\right),\left(n_{r}=1 ; m=m_{0}-2\right)$, $\left(n_{r}=2 ; m=m_{0}-4\right)$, etc. Within the subspace of these degenerate states the matrix elements of $\Delta H$ are all zero, except the ones linking neighboring states, and they are of order $\varepsilon$. Therefore the problem of diagonalizing $H+\Delta H$ is equivalent to a tight-binding model, with a position-dependent hopping integral connecting only nearest neighbors. The correction $\Delta \epsilon$ to the singleparticle energies due to $\Delta H$ is thus of order $\varepsilon$, and the energy of the single-particle states is

$$
\epsilon_{n_{r}, m}^{\prime}=\epsilon_{n_{r}, m}+\Delta \epsilon=\left[m_{0}+1+\mathcal{O}\left(\varepsilon_{n_{r}, m}\right)\right] \hbar \omega_{\perp},
$$

where $\mathcal{O}\left(\varepsilon_{n_{r}, m}\right)$ denotes a term of order $\varepsilon$ which depends on $n_{r}$ and $m$. The corresponding single-particle states are given by

$$
\phi_{n_{r}, m}^{\prime}=\phi_{n_{r}, m}+\sum_{n_{r}^{\prime}, m^{\prime}} \mathcal{O}\left(\varepsilon_{n_{r}^{\prime}, m^{\prime}}\right) \phi_{n_{r}^{\prime}, m^{\prime}}
$$

where $n_{r}^{\prime}$ and $m^{\prime}$ can take all the possible values, with the exception $n_{r}^{\prime} \neq n_{r}$, and $m^{\prime} \neq m$. The results of Eqs. (6) and (7) can also be seen by expanding Eqs. (4) and (5) respectively in powers of $\varepsilon$.

To be more explicit, the wavefunctions we have to consider for the lowest-energy state of the system are dominated by the component having no radial nodes,

$$
\phi_{0, m}^{\prime}=\phi_{0, m}+\sum_{n_{r}^{\prime}, m^{\prime}} \mathcal{O}\left(\varepsilon_{0, m^{\prime}}\right) \phi_{n_{r}^{\prime}, m^{\prime}},
$$

with $n_{r}^{\prime}=1,2, \ldots$, and $m^{\prime}=m-2, m-4, \ldots$

Equation (6) implies that the lowest energy of $N$ (noninteracting) bosons having $L$ units of angular momentum (measured relative to that of the ground state) is

$$
E(L, N)=\left[L+N \mathcal{O}\left(\varepsilon_{L, N}\right)\right] \hbar \omega_{\perp},
$$

where $\mathcal{O}\left(\varepsilon_{L, N}\right)$ is a term of order $\varepsilon$ that depends on $L$ and $N$. According to Eq. (9) there is a spreading in the energy of the states around the value $L \hbar \omega_{\perp}$, which is of order $N \varepsilon \hbar \omega_{\perp}$. As long as $\varepsilon$ is small, $\varepsilon \ll 1$, the effect of this term is not substantial for $L$ being $\mathcal{O}(N)$.

\section{Effect of interactions - Hierarchy of terms}

Turning to the effect of the interactions between the atoms, we assume that these are of zero range,

$$
V_{\mathrm{int}}=\frac{1}{2} U_{0} \sum_{i \neq j} \delta\left(\mathbf{r}_{i}-\mathbf{r}_{j}\right)
$$

where $U_{0}=4 \pi \hbar^{2} a_{\mathrm{sc}} / M$ is the strength of the effective two-body potential, with $a_{\text {sc }}$ being the scattering length for atom-atom collisions. In the present article we examine only the case of effective repulsive interactions between the atoms, $a_{\mathrm{sc}}>0$.

In the limit that the interactions are weak, the energy of the states which are degenerate in the absence of interactions is spread over a width of order $n U_{0}$, where $n$ is the atom density and thus the following condition has to be satisfied

$$
n U_{0} \ll \hbar \omega_{\perp} .
$$

If $N$ is the number of atoms in the trap, and assuming that the gas is trapped along the $z$ axis on a length scale of order $R_{z}$, and in the transverse direction on a length scale of order $a_{\perp}, n$ is of order $N / a_{\perp}^{2} R_{z}$; the above condition can then be written as

$$
v_{0} \ll \hbar \omega_{\perp} / N \text {, }
$$

where $v_{0} \sim U_{0} / a_{\perp}^{2} R_{z}$. Equation (12) implies that the scattering length has to scale as

$$
a_{\mathrm{sc}} \sim \frac{R_{z}}{N}
$$


for the assumption of weak interactions to be valid (for fixed $\omega_{\perp}$.) As a result, the ratio $\varepsilon \hbar \omega_{\perp} / N v_{0}$ is $\ll 1$, and therefore

$$
\varepsilon \hbar \omega_{\perp} \ll N v_{0} \ll \hbar \omega_{\perp} .
$$

Under the above conditions the many-body states built up by $\phi_{0, m}^{\prime}$ are quasi-degenerate. Therefore one has to deal with a problem similar to that of Refs. 6 13 of a symmetric confining potential, with a set of (quasi)degenerate states whose degeneracy is lifted by the interactions. Under the conditions given by Eq. (14), the energy of the lowest state (the so-called yrast state) with $L$ units of angular momentum and $N$ particles is given by

$$
\mathcal{E}(L, N)=E(L, N)+E_{\text {int }}(L, N) .
$$

Here $E(L, N)$ is given by Eq. (9), and we write it for convenience as $E(L, N)=(L+a N \varepsilon) \hbar \omega_{\perp}$, where $a$ is a parameter of order unity that depends on $L$ and $N$. Also $E_{\text {int }}(L, N)$ is the interaction energy due to $V_{\text {int }}$. For $\varepsilon=0, E_{\text {int }}(L, N)$ has been expressed as a power series in $N$ and $L$ in Ref. 113,

$E_{\text {int }}(L, N)=\left(b_{1} N^{2}+b_{2} N L+b_{3} N+b_{4} L+\ldots\right) v_{0}$,

where $b_{i}$ are parameters of order unity that depend on $L$ and $N$. For $\varepsilon \neq 0, E_{\text {int }}(L, N)$ becomes, to leading order in $\varepsilon$,

$E_{\text {int }}(L, N)=(1+c \varepsilon)\left(b_{1} N^{2}+b_{2} N L+b_{3} N+b_{4} L+\ldots\right) v_{0}$,

where $c$ is a a parameter of order unity that depends on $L$ and $N$. The terms proportional to $c \varepsilon$ result from the mixing of states with $n_{r} \neq 0$ (to leading order), but they are not of importance in our problem.

According to the above equations $\mathcal{E}(L, N)$ is given by (to leading order)

$$
\mathcal{E}(L, N)=L \hbar \omega_{\perp}+\left(b_{1} N+b_{2} L\right) N v_{0}+a N \varepsilon \hbar \omega_{\perp}+\ldots,
$$

with $L \hbar \omega_{\perp} \gg N^{2} v_{0} \gg N \varepsilon \hbar \omega_{\perp}$, so the terms of order $N^{2} v_{0}$ in the interaction energy dominate the term in the energy due to the symmetry-breaking potential. For a given $L$, the corresponding angular frequency of rotation $\Omega$ of the gas is

$$
\Omega=\frac{1}{\hbar} \frac{\partial \mathcal{E}(L, N)}{\partial L} .
$$

The single-particle density contours that we derive in Sec. III refer to a frame that rotates with this frequency $\Omega$, which we assume is also the frequency of rotation $\Omega_{0}$ of the asymmetric potential $\Delta H$ (under equilibrium conditions.) In Sec. IV we examine the response of the gas if the trap stops to rotate, i.e., if $\Omega_{0} \rightarrow 0$.

\section{Connection with experiment}

Before we proceed, we explore the relevance of our study to experiment. As we mentioned in the Introduction, in the experiment of Ref. [4], more than 100 vortices were created and as argued in Ref. [16], in such a system one can easily get to the regime of weak interactions.

It is even more interesting that in the recent experiment described in Ref. 24, it has become possible to create Bose-Einstein condensates of ${ }^{23} \mathrm{Na}$ atoms in cigar-shaped traps, where transversely to the long axis of the trap the gas is in the lowest harmonic-oscillator level. More specifically, the trapping frequencies used were $\omega_{z} / 2 \pi=3.5 \mathrm{~Hz}$ and $\omega_{\perp} / 2 \pi=360 \mathrm{~Hz}$, which implies that $a_{z} \approx 11 \mu \mathrm{m}$ and $a_{\perp} \approx 1 \mu \mathrm{m}$. To put as many atoms as possible, Görlitz et al. created a Bose-Einstein condensate which was in the Thomas-Fermi regime of strong interactions along the long axis, but on the other hand it was in the lowest harmonic-oscillator level perpendicular to the long axis. Under these conditions, the transverse width of the cloud is on the order of the oscillator length $a_{\perp}$, and for the interaction energy to be comparable to $\hbar \omega_{\perp}$,

$$
N_{c} \sim \frac{R_{z}}{a_{\mathrm{sc}}},
$$

where $R_{z}$ is the width of the cloud along the $z$ axis. As we mentioned, the cloud is in the Thomas-Fermi limit along this direction, and therefore

$$
\frac{R_{z}}{a_{z}} \approx\left(\frac{N a_{\mathrm{sc}} a_{z}}{a_{\perp}^{2}}\right)^{1 / 3} .
$$

From Eqs. 20 and (21) we find that

$$
N_{c} \sim \frac{a_{z}^{2}}{a_{\perp} a_{\mathrm{sc}}},
$$

which gives $N_{c} \approx 5 \times 10^{4}$. Indeed, it was confirmed that as $N$ varied around this value, the cloud passed from the limit of strong interactions to the one of weak interactions.

Under the above conditions, the coherence length $\xi$ at the center of the cloud is

$$
\frac{\xi}{a_{\perp}} \sim\left(\frac{a_{z}^{2}}{N a_{\mathrm{sc}} a_{\perp}}\right)^{1 / 3} \sim\left(\frac{N_{c}}{N}\right)^{1 / 3} .
$$

For $N \ll N_{c}, a_{\perp} \ll \xi$, i.e., the coherence length becomes larger than the size of the trap and the properties of the system under rotation resemble those of superfluid nuclei.

\section{DENSITY CONTOURS}

We investigate now the (normalized) single-particle density of the atoms $\rho(\mathbf{r})$, in the many-body state $\left|\Psi_{L, N}\right\rangle$ with $N$ atoms and $L$ units of angular momentum, 


$$
\rho(\mathbf{r})=\frac{1}{N}\left\langle\Psi_{L, N}\left|\sum_{i} \delta\left(\mathbf{r}-\mathbf{r}_{i}\right)\right| \Psi_{L, N}\right\rangle .
$$

From the exact solutions that come from diagonalization of $V_{\text {int }}$ in the subspace of degenerate states [6.9, the single-particle density is always axially symmetric, reflecting the symmetry of the confining potential.

Let us now introduce a mean-field wavefunction [7, 12], which is a Fock state expressed as a product of singleparticle states:

$$
\Psi_{L, N}\left(\mathbf{r}_{1}, \mathbf{r}_{2}, \ldots, \mathbf{r}_{N}\right)=\psi\left(\mathbf{r}_{1}\right) \times \psi\left(\mathbf{r}_{2}\right) \ldots \psi\left(\mathbf{r}_{N}\right) .
$$

The single-particle states $\psi\left(\mathbf{r}_{i}\right)$ can be expanded on the basis of the harmonic-oscillator eigenstates $\phi_{0, m}\left(\mathbf{r}_{i}\right)$ with no radial nodes and an angular momentum $m \hbar$ along the axis of rotation:

$$
\psi\left(\mathbf{r}_{i}\right)=\sum_{m=0}^{\infty} c_{m} \phi_{0, m}\left(\mathbf{r}_{i}\right) .
$$

In the approach of Refs. [7] the coefficients $c_{m}$ in Eq. (26) are treated as variational parameters with two constraints imposed on them: the first is the normalization condition, $\sum_{m}\left|c_{m}^{2}\right|=1$, and the second is that the expectation value of the angular momentum be equal to $l=L / N, \sum_{m} m\left|c_{m}\right|^{2}=L / N$. One then calulates the interaction energy which is

$$
E_{\text {int }}(L, N)=\frac{1}{2} N^{2} v_{0} \int|\psi(\mathbf{r})|^{4} d^{3} r
$$

to leading order in $N$, and minimizes it with respect to the $c_{m}$. As a result of this approach, for repulsive interactions and within the mean-field approximation a vortex array develops as $L / N$ increases [7, 12].

The mean-field wavefunction above has the following features or advantages: (i) it gives the exact leadingorder term of the interaction energy, i.e., the $N^{2} v_{0}$ [13], (ii) the corresponding single-particle density breaks the rotational symmetry, since the single-particle states given by Eq. (26) have some axes of symmetry [7] 12] by construction, and (iii) it is essentially a one-body wavefunction, and thus it is easy to work with and to visualize. As shown in Ref. [12] there is a freedom in one of the phases of the coefficients $c_{m}$ reflecting the rotational invariance of the confining potential. Therefore even in the mean-field approximation one can construct a circularlysymmetric state by superimposing the above states corresponding to different angles, and therefore to different orientations.

However, even an infinitesimally small $\varepsilon$ is enough to change $\rho(\mathbf{r})$ from being symmetric, looking like the one given by mean-field, and therefore with a specific orientation. To see this more clearly, we notice that the leading-order $N^{2} v_{0}$ term in the interaction energy given by the mean-field approximation is identical to the one that comes from the exact solution, as shown in Ref. [13]. For any nonzero value of $\varepsilon$ the term of order $N \varepsilon \hbar \omega_{\perp}$ has to become as small as possible, as Eq. (18) implies, in addition to the leading-order term of the interaction energy, i.e., the $N^{2} v_{0}$. In order to minimize the $N \varepsilon \hbar \omega_{\perp}$ term, the phase that is free to take any value when $\varepsilon=0$ (reflecting the rotational invariance of the confining potential) has to be chosen appropriately. Therefore for $\varepsilon \neq 0$ there will be in general some preferred orientations of the cloud corresponding to some (distinct) values of the phase which minimize the $N \varepsilon \hbar \omega_{\perp}$ term. These axes of symmetry rotate with an angular frequency $\Omega$ given by Eq. (19).

Therefore, according to our analysis, an infinitesimally asymmetric confining potential makes the circularly symmetric single-particle density of the cloud unstable against the formation of a vortex array as given by the mean-field approximation. This array of vortices has been investigated in Refs. [7] 12] (see Fig. 2 in Ref. (7] and Figs. 3 and 4 in Ref. [12]).

\section{TIMESCALES}

Related to the above remarks are some timescales which we examine now. We consider a problem corresponding to the experiment of Ref. [3], where the atoms get their angular momentum when they are still in the normal phase, above the transition temperature, by rotating $\Delta H$ with some angular frequency $\Omega(t)$, which slowly approaches the value $\Omega_{0}$. The gas is then cooled down to the condensed phase, in the presence of the external rotating drive. The system equilibrates in the rotating frame, and eventually $\Delta H$ is turned off slowly. After some time the atoms are released from the trap and expand, and their density is observed. Since the whole process occurs adiabatically, the state of the system is determined by minimizing the energy in the rotating frame, i.e., by minimizing $\mathcal{E}^{\prime}(L, N)=\mathcal{E}(L, N)-L \hbar \Omega_{0}$.

Therefore initially, when the gas is in the normal phase, it has some angular momentum, which changes during the cooling process to the condensed phase. When the cooling process is completed the gas is not in equilibrium yet, since its angular frequency of rotation $\Omega$ given by Eq. (19) is not necessarily equal to the frequency of rotation of the trap $\Omega_{0}$. One therefore has to wait for some time for the system to equilibrate, so that $\Omega$ will become equal to $\Omega_{0}$. After this time interval the gas is driven by the rotating asymmetric trap and it is in a "dynamical" steady state.

The interesting question is how does the gas respond if the symmetry-breaking potential stops to rotate. If this process occurs adiabatically, i.e., on timescales much larger that $\omega_{\perp}^{-1}$, the gas will go back to rest. The opposite limit, when the trap stops to rotate on a timescale that is short as compared to $\omega_{\perp}^{-1}$, is non-trivial, however. In this case the gas experiences a time-dependent potential. This potential induces transitions to states with a different angular momentum. Because of the symmetry of 
the perturbation $\Delta H$ we have chosen (i.e., $x^{2}-y^{2}$ ), only states which differ by two units of angular momentum are linked via $\Delta H$. The rate $\Gamma$ for the system to lose two units of angular momentum (due to the torque coming from $\Delta H$ that no longer drives the gas) is of order

$$
\Gamma=\frac{2 \pi}{\hbar} \frac{\left|\left\langle\Phi_{L-2, N}|\Delta H| \Psi_{L, N}\right\rangle\right|^{2}}{|\Delta \mathcal{E}(L, N)|} .
$$

Here $\left|\Phi_{L-2, N}\right\rangle$ is the state that couples to the ground state $\left|\Psi_{L, N}\right\rangle$ via $\Delta H$, and $\Delta \mathcal{E}(L, N)$ is the energy difference between them. The rate $\Gamma$ is dominated by the transition to the state that is closest in energy to the yrast. Since the yrast state consists mostly of singleparticle states with no radial excitations, the state that is closest in energy is the one dominated by single-particle states with one unit of radial excitations. The energy denominator in Eq. (28) is, to leading order,

$$
|\Delta \mathcal{E}(L, N)| \sim N v_{0},
$$

plus corrections of order $N \varepsilon \hbar \omega_{\perp}$.

To proceed to the calculation of the matrix element of Eq. (28), let us denote as $N_{m}$ the occupancy of the state of the harmonic oscillator $\phi_{0, m}$ when the system is in the yrast state $\left|\Psi_{L, N}\right\rangle$. Within the mean-field approach $N_{m}=N\left|c_{m}\right|^{2}$, whereas within the exact approach one needs to project the yrast state on the basis $\phi_{0, m}$ and get the corresponding $N_{m}$. There is very strong evidence that the two approaches give the same result for $N_{m}$ 13,25]. The above matrix element is on the order of

$$
\left\langle\Phi_{L-2, N}|\Delta H| \Psi_{L, N}\right\rangle \sim \varepsilon \hbar \omega_{\perp} \sum_{m=0}^{\infty} \sqrt{N_{m} N_{m+2}} .
$$

Typically $m$ in the above sum runs over a few states only, since states with high values of $m$ are not occupied. Now two possibilities need to be considered. Depending on the value of the ratio $L / N$, the sum on the right of Eq. (30) can be either of order $N^{1 / 2}$, or of order $N$ [7, 12, 13 and $\Gamma$ depends crucially on that. We examine each case separately below.

\section{A. Fast relaxation}

When the yrast state consists of at least two states $N_{m}$ and $N_{m+2}$ which are of order $N$, the matrix element of Eq. (30) is of order $N \varepsilon \hbar \omega_{\perp}$ and from Eqs. (28) and (29) we get that the rate for the system to lose $L \sim N$ units of angular momentum is

$$
\frac{\Gamma}{N}=\omega_{\perp} \varepsilon^{2} \frac{\hbar \omega_{\perp}}{v_{0}} \chi(L / N),
$$

where $\chi(L / N)$ is a function of $L / N$, and its value is of order unity [26].
An important conclusion resulting from Eq. (31) is that the timescale for relaxation of angular momentum can get long as compared to the period of the trap, provided that

$$
\varepsilon \ll\left(\frac{v_{0}}{\hbar \omega_{\perp}}\right)^{1 / 2} .
$$

Since, according to Eq. (12) $v_{0} \ll \hbar \omega_{\perp} / N$ for the assumption of weak interaction to be valid, therefore

$$
\varepsilon \propto \frac{1}{N^{1 / 2}}
$$

for the decay rate to be $\ll \omega_{\perp}$. Under the conditions of Ref. [24], $\varepsilon \ll 0.01$ for $N=5 \times 10^{4}$, which is a rather restrictive condition. Realistic symmetric traps have anisotropies on the order of $1 \%$ [27], and therefore the relaxation rate of angular momentum should be on the order of the trap frequency, even for the least asymmetric traps.

The above case of fast relaxation applies to all the yrast states with $L / N \leq 2.03$, except for the region around the single vortex, $L / N \approx 1[12$. Above the value $L / N \approx 2.03$ the system shows a discontiuous transition from a twofold symmetric state to a three-fold symmetric state [12]. Also for $L / N \approx 1$ the yrast state is dominated by the occupancy of a single state, the $m=1$ [6 8, 12]. These cases are examined in the following subsection.

\section{B. Slow relaxation}

For $L / N \gtrsim 2.03$, the yrast state is three-fold symmetric; therefore for $L / N \gtrsim 2.03$ and for $L / N \approx 1$, the matrix element of Eq. (30) is instead given by

$$
\left\langle\Phi_{L-2, N}|\Delta H| \Psi_{L, N}\right\rangle \sim \sqrt{N} \varepsilon \hbar \omega_{\perp} .
$$

In this case,

$$
\frac{\Gamma}{N} \sim \omega_{\perp} \varepsilon^{2} \frac{\hbar \omega_{\perp}}{N v_{0}} .
$$

From Eq. 35) one sees that for the state to be stable,

$$
\varepsilon \ll\left(\frac{N v_{0}}{\hbar \omega_{\perp}}\right)^{1 / 2},
$$

which is a condition much easier to satisfy, since although $\varepsilon$ has to be numerically small, it does not have to scale with the number of atoms, $N$.

To summarize the results of this section, the presence of $\Delta H$ that rotates with an angular frequency $\Omega_{0}$ results in (i) transferring angular momentum, and (ii) favouring the creation of a vortex array instead of a circularlysymmetric ground state. Finally stopping the rotation of $\Delta H$ results in a relaxation rate of the angular momentum of the gas that depends on the yrast state considered, i.e., on the ratio $L / N$. For $L / N \leq 2.03$, except $L / N \approx 1$, the 
rate is low as long as $\varepsilon \sim N^{-1 / 2}$ and the states are stable under such weak perturbations. For $L / N \approx 1$, and for $L / N \gtrsim 2.03$, the relaxation rate is suppressed by a factor of $1 / N$, and $\varepsilon$ has to be numerically small, but independent of $N$, for these states to be stable.

The relaxation rate can also be evaluated for higher values of $L / N$. According to Ref. 7], these states have certain symmetry, and as long as this symmetry is not two-fold [28], the relaxation rate is given by Eq. (35).

\section{CONCLUSIONS}

In conclusion, we have investigated the effect of a small asymmetry in the confining potential of a weakly-interacting Bose-Einstein condensate, which rotates bringing the gas into rotation. Three important results have come out of this study: (i) the limit of weak interactions can be achieved, even under current experimental conditions, (ii) even in a weakly-asymmetric rotating trap, in its lowest state the system develops a vortex array for an effective repulsive interaction between the atoms, and (iii) if the gas is no longer driven externally, the rate of relaxation of the angular momentum depends on the yrast state considered. For the specific form of the perturbation $\Delta H$ (i.e., $x^{2}-y^{2}$ ), if the yrast state does not have some symmetry, or if it has a two-fold symmetry, the rate of relaxation of angular momentum is large. On the other hand, the unit vortex, or states with $s$-fold symmetry, with $s \geq 3$ are expected to be more robust against anisotropies of the confining potential, since the rate of relaxation of angular momentum is lower by a factor of $1 / N$. These are definite theoretical predictions and it is interesting to confirm them experimentally after the expected creation of vortices in weakly-interacting Bose-Einstein condensates.

\section{ACKNOWLEDGMENTS}

I am grateful to A. Jackson, B. Mottelson, and S. M. Reimann for useful discussions.

[1] F. Dalfovo, S. Giorgini, L. P. Pitaevskii, and S. Stringari, Rev. Mod. Phys. 71, 463 (1999).

[2] M. R. Matthews, B. P. Anderson, P. C. Haljan, D. S. Hall, C. E. Wieman, and E. A. Cornell, Phys. Rev. Lett. 83, 2498 (1999).

[3] K. W. Madison, F. Chevy, W. Wohlleben, and J. Dalibard, Phys. Rev. Lett. 84, 806 (2000); J. Mod. Opt. 47, 2715 (2000).

[4] J. R. Abo-Shaeer, C. Raman, J. M. Vogels, and W. Ketterle, Science 292, 476 (2001).
[5] P. C. Haljan, I. Coddington, P. Engels, and E. A. Cornell, e-print cond-mat/0106362.

[6] N. K. Wilkin, J. M. F. Gunn, and R. A. Smith, Phys. Rev. Lett. 80, 2265 (1998).

[7] D. A. Butts and D. S. Rokhsar, Nature (London) 397, 327 (1999).

[8] B. Mottelson, Phys. Rev. Lett. 83, 2695 (1999).

[9] G. F. Bertsch and T. Papenbrock, Phys. Rev. Lett. 83, 5412 (1999).

[10] N. K. Wilkin and J. M. F. Gunn, Phys. Rev. Lett. 84, 6 (2000).

[11] M. Linn and A. L. Fetter, Phys. Rev. A 60, 4910 (1999).

[12] G. M. Kavoulakis, B. Mottelson, and C. J. Pethick, Phys. Rev. A 62, 63605 (2000).

[13] A. D. Jackson, G. M. Kavoulakis, B. Mottelson, and S. M. Reimann, Phys. Rev. Lett. 86, 945 (2001).

[14] X. Liu, H. Hu, L. Chang, W. Zhang, S.-Q. Li, and Y.-Z. Wang, Phys. Rev. Lett. 87, 030404 (2001).

[15] M. Linn, M. Niemeyer, and A. L. Fetter, Phys. Rev. A 64, 023602 (2001).

[16] Tin-Lun Ho, Phys. Rev. Lett. 87, 060403 (2001).

[17] D. S. Rokhsar, Phys. Rev. Lett. 79, 2164 (1997).

[18] A. A. Svidzinsky and A. L. Fetter, Phys. Rev. Lett. 84, 5919 (2000).

[19] E. Lundh, C. J. Pethick, and H. Smith, Phys. Rev. A 58, 4816 (1998).

[20] J. J. García-Ripoll and V. M. Pérez-García, Phys. Rev. A 60, 4864 (1999).

[21] D. L. Feder, C. W. Clark, and B. I. Schneider, Phys. Rev. A 61, 011601(R) (1999); Phys. Rev. Lett. 82, 4956 (1999).

[22] E. Lundh and P. Ao, Phys. Rev. A 61, 063612 (2000).

[23] E. Lundh, e-print cond-mat/0103272.

[24] A. Görlitz, J. M. Vogels, A. E. Leanhardt, C. Raman, T. L. Gustavson, J. R. Abo-Shaeer, A. P. Chikkatur, S. Gupta, S. Inouye, T. P. Rosenband, D. E. Pritchard, and W. Ketterle, Phys. Rev. Lett. 87, 130402 (2001).

[25] G. M. Kavoulakis, B. Mottelson, and S. M. Reimann, e-print cond-mat/0109329.

[26] For a noninteracting gas, $v_{0}=0$, the denominator in the above equation $|\Delta \mathcal{E}(L, N)|$ is $\sim N \varepsilon \hbar \omega_{\perp}$, (which was previously neglected because of the condition $N \varepsilon \hbar \omega_{\perp} \ll$ $\left.N v_{0}\right)$, and $\Gamma / N \sim|\varepsilon| \omega_{\perp}$.

[27] J. Dalibard, private communication.

[28] We note that for an $s$-fold symmetric state, only the states $N_{m}$ are macroscopically occupied, where $m=\alpha s$ with $\alpha=0,1,2, \ldots$. 